'División de Anestesiología. Programa de Estudios Médicos Humanísticos, Facultad de Medicina. Pontificia Universidad Católica de Chile. Santiago, Chile.

Trabajo no recibió financiamiento.

El autor declara no tener conflictos de interés.

Recibido el 27 de junio de 2018, aceptado el 5 de diciembre de 2018.

Correspondencia a: Dr. Jorge Dagnino-Sepúlveda San Crescente 269-Las Condes. Santiago, Chile. jorge.dagnino@gmail.com

\section{William Hunter (1718-1783): su legado a trescientos años de su nacimiento}

\author{
JORGE DAGNINO-SEPÚLVEDA ${ }^{1}$
}

\section{William Hunter (1718-1783): his legacy three hundred years from his birthday}

The aim of this paper is to evaluate some aspects of the life of William Hunter (1718-1783), and to argue that he deserves a better place in history as he was one of the most outstanding figures in British medicine of the $18^{\text {th }}$ century. A Scotsman, Presbyterian, from a family without means or connections, he underwent a classic education at the University of Glasgow, completed with apprenticeships with William Cullen and Alexander Monro first in Scotland, and then in London, with William Smellie, James Douglas and James Wilkie, in anatomy, obstetrics and surgery, respectively. Despite initial disadvantages, he was highly successful as an anatomist, educator, surgeon, man-midwife, artist, gentleman and collector. He moved and had influences in the highest medical, cultural and social circles of his time, was named Physician Extraordinary to Queen Charlotte, and was a member of many scientific, medical and cultural societies in Great Britain and in France, such as the Royal Society, the Royal College of Physicians and the Societé Royale de Médicine of Paris. His museum was notable in its magnitude and its diversity, including anatomical preparations, coins, shells, plants, birds, insects, fossils, and minerals. He donated his great museum to the University of Glasgow. His figure has been relegated mainly due to the absence of heirs, individuals or institutional, that could have cared for, maintained, and increased his legacy as happened with his brother John.

(Rev Med Chile 2019; 147: 96-102)

Key words: Anatomy; Education, Medical; History, $18^{\text {th }}$ Century; History of Medicine; Museums.
E ste trabajo relata aspectos de la vida de William Hunter, sin duda, una de las figuras más destacadas de la medicina británica en el siglo XVIII, para relevar sus aportes y argumentar que merece un mayor reconocimiento en la historia del que ha gozado hasta ahora.

Nació cerca de Glasgow, el séptimo de diez hermanos y diez años mayor que el último, el segundo John, por lejos el más famoso ${ }^{1,2}$. A los trece años fue a estudiar por cinco años un currículo clásico a la Universidad de Glasgow; entre grandes profesores, lo impresionaría Francis Hutchenson, uno de los fundadores del Enlightenment escocés, pues urgía a sus alumnos "a pensar por ellos mis- mos, despertando en ellos una apreciación de la literatura, artes, y todo lo ornamental y útil en la vida humana, y les imbuía un ansia por el conocimiento y un espíritu inquisitivo"2.

Después de regresar a casa y sin trabajo, aceptó ser aprendiz de William Cullen, médico amigo de la familia en la vecina Hamilton. El aprendizaje era entonces una de las principales vías de formación, especialmente para los cirujanos ${ }^{3-5}$. Durante los siguientes tres años, que después recordaría los más felices, la relación con Cullen progresó a una amistad que mantendrían por toda la vida. William diría de Cullen "a quien más debo y más quiero de todos los hombres del mundo"; Cullen 
lo describiría "un joven cuya conversación era notablemente vivaz y agradable, y toda su conducta era más estricta y constantemente correcta que la de cualquier otro" ${ }^{2}$. Le ofreció asociarse para abordar la cirugía y la obstetricia, por lo que, para completar su formación, fue a Edinburgo donde, entre otros cursos, asistió al de anatomía de Alexander Monro primus; estos cursos particulares eran populares, substituían carencias de la formación universitaria y constituían otro de los recursos habituales en la formación médica ${ }^{4,5}$. Monro fue uno de los fundadores de la Escuela de Medicina de Edinburgo, que luego alcanzaría gran prestigio, con Cullen como uno de sus más famosos profesores. Hunter juzgó el curso de Monro bueno, pero incompleto, agregando que "aprendí más escuchando que viendo"2.

Viajó a Londres para seguir su formación con William Smellie, un prestigiado obstetra, entonces llamados accoucheur o man-midwife; el término obstetra, de origen griego, recién empezó a ser usado en el sentido actual en el siglo XIX. Durante este período, se perfeccionó en filosofía experimental con Desaguliers, anatomía con Nicholls, y cirugía con Wilkie, en el recién fundado hospital de St. George. Todos eran figuras prestigiosas y muestran la inquietud de Hunter por lograr la mejor formación posible; el curso con Nicholls reforzó la impresión sobre lo limitado de la cobertura y de la experiencia práctica.

El siguiente paso fue un aprendizaje con James Douglas, el del saco, un médico y obstetra respetado, culto y rico; dada la educación clásica de Hunter, el arreglo incluía la tutoría del hijo de Douglas. Poco después falleció su mentor, quien había pedido respetar el acuerdo que incluía un viaje de estudios con su hijo a París. Allá, Hunter tomó otros cursos de anatomía en los que notó la diferencia que significaba disponer de cadáveres para lograr una experiencia práctica; a su regreso en 1744 , a los veintiseis años, Hunter había terminado una formación excepcional e iniciaba una exitosa carrera que parece mejor abordar en áreas para mejor aquilatar su progreso (Figura 1).

\section{Anatomista}

Fue descrito como el "padre de la Anatomía de este Reino y, en algún grado, de todo el mundo",, 7 . Buena parte de su tiempo lo dedicaba a la disec- ción y a la preparación de muestras anatómicas para sus demostraciones. No siempre publicó sus observaciones, ya que eran una atracción para sus cursos; esta práctica propició conflictos de prioridad, con sus ayudantes o con terceros, siendo las más comentadas aquella con Monro secundus sobre el sistema linfático, con Pott por las hernias congénitas y con su hermano John por la circulación placentaria ${ }^{2}$. Estas disputas han sido interpretadas negativamente por algunos, como si su repetición implicase culpabilidad, pero la evidencia ponderada da la razón a William² ${ }^{2}$; además, hizo numerosas presentaciones de los aportes de ayudantes o alumnos, incluyendo a John, lo que habla en contra de una conducta de apropiación de aportes ajenos ${ }^{2}$. El conflicto de prioridades subyacería al distanciamiento con su hermano, aunque hay otras explicaciones plausibles como los celos, diferencias de carácter o discusiones de dinero ${ }^{2,7}$.

Entre sus muchos aportes figura la descripción normal y patológica del cartílago articular, la extensión y función del sistema linfático, y la circulación placentaria ${ }^{7,9}$. Fue miembro de la Royal Society y distinguido como miembro extranjero de las parisinas Académie Royale des Sciences, donde ocupó el sillón vacante de Malpighi y von Haller,

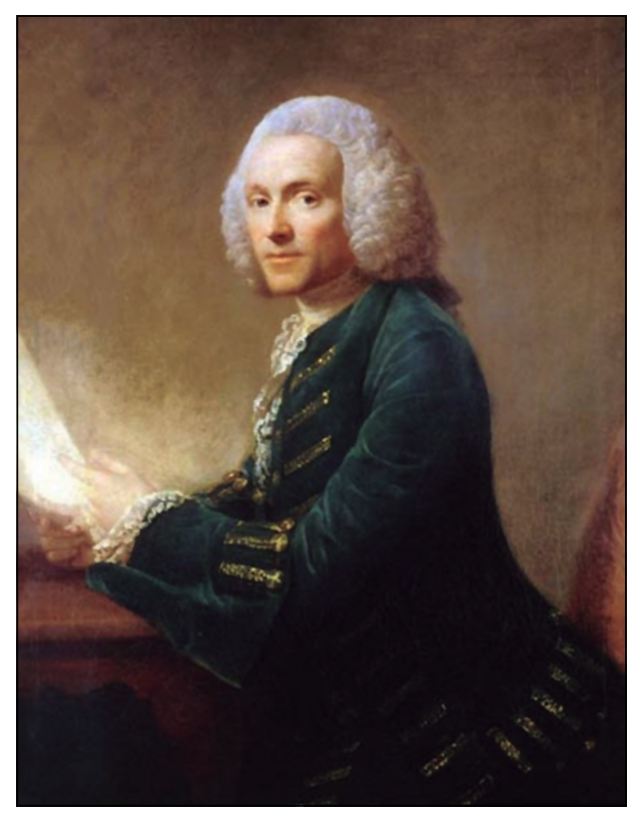

Figura 1. William Hunter, retrato por Allan Ramsay (17131784), óleo sobre tela c.1764-5. CAntonia Reeve and The Hunterian, University of Glasgow 2018. 
y de la Societé Royale de Médicine. Su obra magna, Anatomy of the Human Gravid Uterus/Anatomia uteri humani gravidi, parece mejor comentarla como arte aunque fue una contribución anatómica mayor ${ }^{10}$.

\section{Empresario}

Muchos de sus logros pueden ser entendidos desde la perspectiva de un empresario en una época donde el emprendimiento fue ampliamente entendido, fomentado y buscado como una oportunidad de obtener dinero, influencia y cambio en la posición social ${ }^{11}$. Hunter identificó la necesidad de ofrecer cursos más completos con experiencia práctica en disección y los desarrolló a la perfección. Su escuela le aportaría grandes recursos, al igual que su práctica médica, pero también fue un inversionista y especulador juicioso, exitoso y afortunado, pues habría ganado la lotería. La evolución de sus finanzas muestra tres saltos cuantiosos que corresponderían a esa suerte y a especulaciones con bonos del gobierno y acciones de la East India Company ${ }^{12}$.

\section{Médico y obstetra}

En 1747 fue admitido a la Corporation of Surgeons, lo que le permitió acceder a nombramientos en los recientemente inaugurados Middlesex Hospital y el British Lying-in Hospital for Married Women que le aseguraron una práctica constante y el acceso a material para sus disecciones y preparaciones anatómicas. Paralelamente, inició su práctica privada donde su personalidad y competencias merecieron una creciente confianza de personas de diversos estratos, incluyendo la alta burguesía y la nobleza, alcanzando gran prestigio y llegando a ser nombrado médico extraordinario de la reina Carlota ${ }^{2,7,11,13}$. La especialidad obstétrica, como todas cuando nacientes, tenía opositores entre aquellos a quienes amenazaba, en este caso las matronas, y el menosprecio de aquellos que la consideraban inferior intelectualmente, los physicians, para quienes usar las manos o tocar directamente a los enfermos y a sus fluidos era despreciable; los cirujanos, a su vez, miraban en menos a los accoucheurs pues la práctica no era medicina, sino esfera de comadronas; $y$ to- dos subestimaban a los apotecarios, a quienes consideraban tenderos ${ }^{14,15}$. En 1750, Glasgow le confirió el grado médico que le permitió ingresar como licenciado al Royal College of Physicians, aunque no como fellow pues solo los graduados de Oxford y Cambridge, que no aceptaban católicos ni disidentes, podían serlo. Más tarde cofundaría la Society of Collegiate Physicians de la que llegaría a ser presidente.

\section{Educador}

Hunter escribió: "adquirir los conocimientos y comunicarlos ha sido el placer, el negocio y la ambición de mi vida" 2 . El anuncio del primer curso en el London Evening Post en 1746 rezaba: "El lunes 1 de febrero a las cinco de la tarde comenzará un curso de Lecciones Anatómicas a las que se agregará las Operaciones de Cirugía con la colocación de Vendajes. Por William Hunter, Cirujano. Los caballeros tendrán una Oportunidad de aprender el Arte de la Disección, durante toda la Temporada Invernal, de la misma manera que en París"16. El curso, en cuatro partes y clases de dos horas de lunes a sábado, era el más completo entre más de una treintena con los que competía y abarcaba, además de anatomía, fisiología, patología y cirugía. Fueron enormemente exitosos y miles de médicos, británicos y extranjeros, lo tomaron durante treinta y siete años; además, asistían artistas y personas curiosas como Adam Smith, Gibbon, Smollett, Burke, Reynolds y Hogarth ${ }^{17-19}$.

En 1748 invitó a su hermano John, que hasta entonces daba tumbos por la vida, como asistente para las disecciones y, quizás más importante entonces, para el procuramiento de cadáveres. La disponibilidad de estos era la principal limitante para la enseñanza de la anatomía en Gran Bretaña. Como en toda época y lugar, las necesidades generan un mercado y, en esto, el negocio de los resurreccionistas, actividad que provocó desconfianzas e incluso revueltas contra los médicos; la situación estalló con el famoso caso de Burke y Hare y las revueltas del cólera en el siglo XIX ${ }^{20-23}$.

En 1762 propuso crear una escuela nacional de Anatomía ofreciendo financiarla si el gobierno ponía el terreno ${ }^{2,7,9}$; como esto no prosperó, construyó en el Great Windmill Street, cercano a Piccadilly, la que sería hasta su muerte casa, sala de disecciones y museo. 
Hay numerosos testimonios que hablan del talento de William como comunicador y formador, infundiendo en sus estudiantes aquello que aprendió de Hutcheson y que fue práctica en su vida profesional y personal: independencia en el pensar, espíritu inquisitivo, rigor, esfuerzo y perseverancia. Ha sido descrito como "el más grande comunicador y profesor que jamás haya existido" ${ }^{2,16}$. Este talento, la provisión estable de cadáveres y la creciente colección de preparaciones "secas y húmedas", explican su enorme éxito en Inglaterra, donde por lejos fue el más reputado, y también internacionalmente, pues revirtió el flujo de estudiantes que hasta entonces iba desde Gran Bretaña al extranjero. Los apuntes de las dos clases inaugurales muestran su erudición, profundidad, claridad y convicciones sobre el método de enseñanza y las responsabilidades suyas y de los alumnos.

Hunter tenía presente la importancia de su influencia como educador: "Uno puede hacer infinitamente más el bien enseñando su arte que practicándolo: los efectos benéficos de esto están centrados en la ventaja de los pocos individuos que se cuidan como pacientes, pero la influencia de un profesor se extiende a toda la nación y desciende a la posteridad"16 (Figura 2).

\section{Artista}

Hunter se relacionó estrechamente con la élite artística y cultural de la época, ${ }^{2,717-19}$. Fue nombrado profesor de Anatomía de la recién fundada Royal Academy en 1767 y socio de la Society of Antiquaries. Su obra más reputada, Anatomia humani uteri gravidi, publicada en 1774 luego de treinta años de trabajo, es sin duda una obra de arte con gran valor anatómico ${ }^{10}$. Hecha con la colaboración del pintor van Rymsdyk y grabada por sir Robert Strange, fue impresa por el mejor de Gran Bretaña, John Baskerville de Birmingham. Mucho se ha discutido sobre la importancia del dibujante, pero es claro que Hunter guió el trabajo, definió los detalles y desarrolló el estilo de las reproducciones que, combinando claridad descriptiva con belleza, es un proceso indudable de creación artística ${ }^{24,25,26}$ (Figura 3).

\section{Coleccionista}

Hubo muchos coleccionistas y colecciones en el siglo XVIII. En Gran Bretaña, la de Sir Hans Sloane, reputadamente el médico más famoso de la primera mitad del siglo, fue comprada por

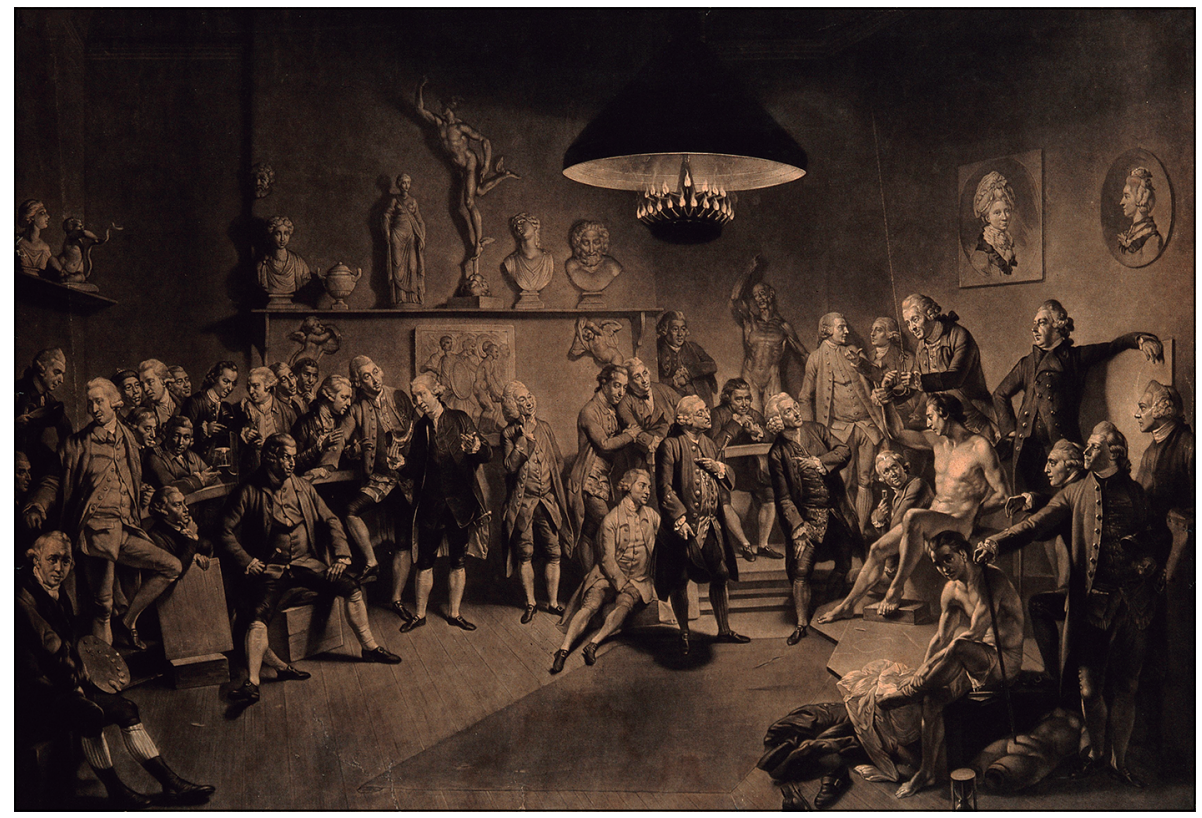

Figura 2. Clase de William Hunter en el Royal College of Arts, 1783, según Johann Zoffany, mediatinta. Wellcome Collection, Creative Commons. 


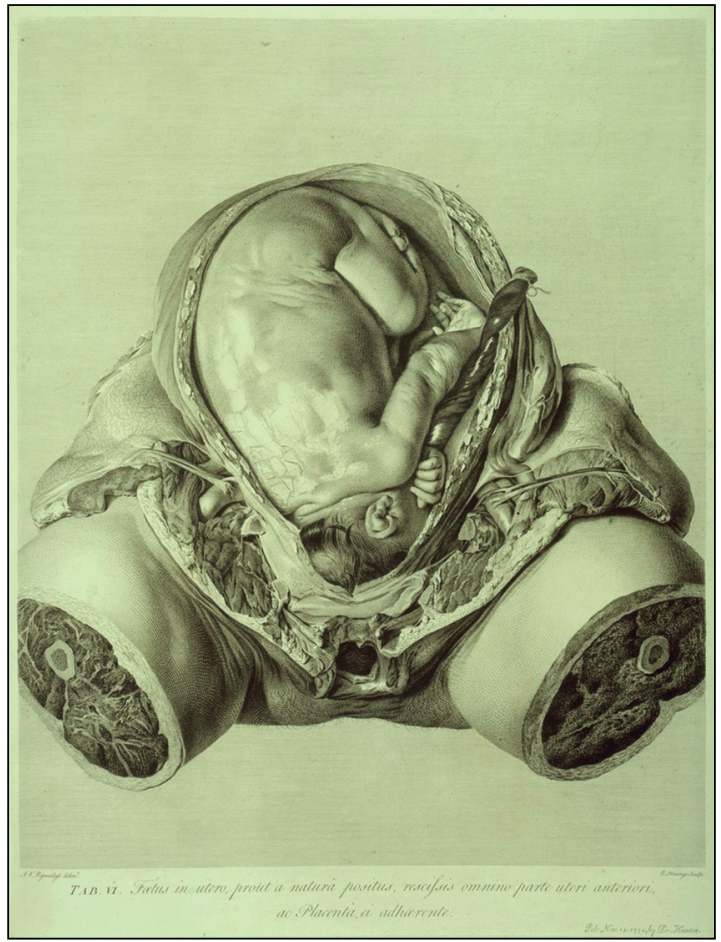

Figura 3. Feto in utero. Placa $N^{\circ} 6$ en Anatomy of the Human Gravid Uterus. Wellcome Images. Creative Commons.

el gobierno a sus sucesores y formó las iniciales del British Museum. La de John Hunter, también comprada por el gobierno y luego entregada al Royal College of Surgeons, pasaría a ser el Hunterian Museum de Londres. La de Richard Mead, rematada luego de su muerte, fue donde William inició sus propias colecciones.

A diferencia de muchos, William era versado en los diferentes aspectos de su interés y en algunos se puede hablar de un erudito. Notoria es su biblioteca con más de diez mil libros, numerosos impresos medievales y contemporáneos, 500 incunables y 6 Caxtons; muchos tienen anotaciones de Hunter en latín o griego ${ }^{2,27}$. Las pinturas destacan por su calidad, su colección de monedas fue la mejor después de la del rey de Francia y aquella de conchas considerada solo después de aquella de la duquesa de Portland. Su colección comprendía también miles de preparaciones anatómicas $y$, entre otras especies, plantas, corales, insectos, pájaros, fósiles y minerales ${ }^{2,27,28}$. Era generoso con su biblioteca y museo pues prestaba y regalaba libros, abría las puertas a estudiosos y guiaba visitas de británicos y extranjeros. Vicq d'Azyr diría "todo elemento [de las colecciones] era, en la mano de Hunter, centro de instrucción e iluminación"2.

Donó sus colecciones a la Universidad de Glasgow junto con el financiamiento para incrementarlas y del edificio para albergarla. Mientras se contruyese, dejó a su sobrino Matthew Baillie el usufructo y cuidado y, a tres custodios el encargo de catalogar, incrementar y preparar las piezas para su traslado a Glasgow. Desgraciadamente, ninguno cumplió con su voluntad. No hubo rendición de cuentas, se perdieron documentos y especies por robo, extravío o simple negligen$\mathrm{cia}^{2,16}$. Muchas piezas y documentos engrosaron el Hunterian de Londres a través de Baillie y también hubo desidia de la Universidad de Glasgow con pérdidas cuantiosas, con material en bodegas sin catalogar por cerca de dos siglos, y con la fragmentación de la colección en diversas dependencias ${ }^{2,16}$. Estos factores y la lejanía explican la desmejorada e injusta posición del Hunterian de Glasgow tras el Hunterian de Londres.

\section{Cabeza de familia}

William pasó a ser el jefe de la familia a los veintitrés años, al morir su padre. Este, aunque no era pobre, era muy cuidadoso con el dinero y William, a pesar de su éxito económico, nunca abandonaría la frugalidad y la sensatez en sus gastos $^{2}$. Le valdría veladas críticas de su sobrino y abierta de su hermano John, quien lo calificó de avaro a pesar de destacar, en otro párrafo, que "donde había sufrimiento real, no acaecido por propia desidia o irresponsabilidad, era muy generoso y de la manera más discreta posible"2,6. Ambos tuvieron motivos para resentir que el pariente rico no les diese más dinero, pero los hechos hablan más bien de alguien pronto a socorrer a quien lo necesitaba pero reacio a hacerlo con quienes, víctimas de sus debilidades de carácter, no lo mereciesen. Es evidente que ayudó a su familia: a su hermano James y también a John, a quien dio empleo y formación, consiguió que fuese aceptado como aprendiz de Cheselden y luego de Pott, arregló para que fuese a Oxford para recibir una educación universitaria, cosa que John desechó apenas dos meses después, a quien ayudó durante su tiempo militar y por diez años después que dejó 
su empleo ${ }^{2}$. Auxilió a su hermana Dorotea al morir la madre, al matrimonio cuando esta desposó a James Baillie, y siguió haciédolo cuando enviudó. Pagó los estudios y le dió una mesada a su sobrino Matthew; dejó a sus sobrinas una cantidad muy considerable y a su sobrino Matthew la propiedad familiar en Escocia y el ususfructo de la escuela en Great Windmill Street y sus colecciones anatómicas, lo que le aseguraba un muy buen pasar para el resto de su vida. Aunque no mencionó a John en sus testamentos, por lo que este trató de anularlos con la cooperación de Baillie y uno de sus albaceas, su hermana Dorotea lo describió así: "de principios en los cuales se podía confiar que haría lo correcto siempre, afectuoso hacia su familia y presto a levantar su mano en defensa de sus hermanos, pero no de esa disposición franca y abierta que hace ser amados a aquellos con principios menos sólidos y afectos más debiles"2.

\section{Gentilhombre}

Hunter partió con evidentes desventajas en la sociedad inglesa del siglo XVIII: escocés, prebiteriano y de una familia con recursos limitados y sin conexiones sociales, culturales o políticas y, además, eligió el camino de la anatomía, de la cirugía y de la obstetricia en una época marcada por la cuna, la religión, la ocupación, el dinero y las conexiones ${ }^{29}$. Al morir era rico, influyente y respetado ampliamente en círculos médicos, culturales y sociales, en Gran Bretaña y en el extranjero, como gentilhombre, anatomista, educador, coleccionista, artista, médico y obstetra.

A pesar de estos logros, que solo pueden ser descritos como extraordinarios, ha sido postergado como individuo, particularmente en la comparación con su evidentemente más famoso hermano John ${ }^{10}$. Una explicación es la carencia de una investigación completa sobre su vida y obra ${ }^{2,11}$. Un obituario $^{30}$ y tres panegíricos luego de su muerte lo recuerdan; uno redactado por quien lo conoció apenas en sus últimos cuatro años, pero con una copia que tiene anotaciones de su hermano John ${ }^{6}$; los otros, de sus pares franceses que lo conocieron solo por reputación, Condorcet y Vicq d'Azyr ${ }^{2}$. Las biografías posteriores son igualmente incompletas, aunque tres son ponderadas en la presentación de los hermanos ${ }^{16,31,32}$. El trabajo de Brock de 30 años es la aproximación más completa que existe y culminó con la edición de su correspondencia en 2008; en ella resaltó la pérdida de muchos de sus documentos, por su propia mano o por sus herederos, hecho que lamentablemente deja en las sombras muchos aspectos de su vida ${ }^{2,6,11}$.

Otra explicación reside en la confusión con su hermano, pues muchos de sus logros han sido atribuidos a John por biógrafos superficiales, y a la sombra de este ${ }^{11,32}$. Aquí se puede argumentar sobre la influencia de factores como los evidentes méritos propios de aquel aunque por sobre estos, quizás, estuvo la necesidad gremial de contar con un héroe para elevar el status de la cirugía como una práctica científica y no solo manual ${ }^{33,34}$. La Hunterian Oration, instituida en 1813, por Baillie y Home, sobrino y cuñado de John respectivamente, y la Hunterian Society en 1819, exaltan hasta hoy, hagiográfica y anualmente la personalidad y aportes de John pero escasamente hablan de William $^{35}$. El Hunterian Museum de Londres, comprado por el gobierno y entregado al Royal College of Surgeons, y acrecentado por generaciones, se constituiría en un lugar de peregrinaje de los cirujanos del mundo; el gratuito Hunterian de Glasgow, a setecientos kilómetros al norte de Londres y las razones ya expuestas, ha tenido un secundario pero orgulloso lugar por más de doscientos años. La especialidad de accoucheur demoraría años en lograr un reconocimiento; el Royal College of Obstetricians recién se fundaría 146 años después de la muerte de William. Así, es evidente que pocos individuos o instituciones se han preocupado de conservar y menos resaltar su legado. Está enterrado en la iglesia de St. James en Piccadilly muy cerca de sus residencias en Jermyn Street y el Great Windmill Street donde terminó su vida; su museo espera justicia, cerca desde donde nació hace trescientos años ${ }^{36}$.

\section{Referencias}

1. Beekman F. Long Calderwood, The Birthplace of the Hunters. Bull NY Acad Med 1943; 19: 849-65.

2. Brock CH. Correspondence of William Hunter, 17401783. London: Pickering \& Chatto, 2008.

3. Lane J. The role of apprenticeship in eighteenth-century England. En Bynum WF y Porter R. William Hunter and the eighteenth-century medical world. Cambridge: Cambridge University Press, 1985.

4. Nutton V, Porter R. (Eds) The History of Medical Educa- 
tion in Britain, Clio Medica. Amsterdam: Rodopi, 1995.

5. Warren MD. Medical Education during the Eighteenth Century. Postgrad Med J 1951; 27: 304-11.

6. Simmons SF, Hunter J. William Hunter 1718-1783. A Memoir. Edited by Brock CH. Glasgow: University of Glasgow Press, 1983.

7. Brock $\mathrm{CH}$. The many facets of Dr. William Hunter (1718-83). Hist Sci 1994; 32: 387-408.

8. Hunter W. On the Structure and Diseases of Articular Cartilage. Phil Trans 1743; 42: 514-21.

9. Hunter W. Two introductory lectures to his last course of Anatomical Lectures to which are added Some Papers relating to Dr. Hunter's intended Plan for establishing a Museum in London. London: J.Johnson, 1784.

10. Hunter W. The Anatomy of the Human Gravid Uterus/ Anatomia Humani Uteri Gravidi. Birmingham: Baskerville, 1774.

11. Porter R. William Hunter: a surgeon and a gentleman. En Bynum WF y Porter R. William Hunter and the eighteenth-century medical world. Cambridge: Cambridge University Press, 1985.

12. Brock CH. The happiness of riches. En Bynum WF y Porter R. William Hunter and the eighteenth-century medical world. Cambridge: Cambridge University Press, 1985.

13. Wilson A. William Hunter and the varieties of manmidwifery. En Bynum WF y Porter R. William Hunter and the eighteenth-century medical world. Cambridge: Cambridge University Press, 1985.

14. Bynum WF. Physicians, hospitals and career structures in eighteenth-century London. En Bynum WF y Porter R. William Hunter and the eighteenth-century medical world. Cambridge: Cambridge University Press, 1985.

15. Hamilton B. The Medical Professions in the Eighteenth Century. The Econom Hist Rev 1951; 4: 141-69.

16. Mather GER. Two great Scotsmen: The brothers William and John Hunter. London: MacMillan, 1893.

17. Kemp M. True to Their Natures: Sir Joshua Reynolds and Dr William Hunter at the Royal Academy. Notes and Records of the Royal Society of London 1992; 46: 77-88.

18. Haslam F. From Hogarth to Rowlandson. Medicine in Art in the Eighteenth Century. Liverpool: Liverpool University Press, 1996.

19. McCormack H. William Hunter and his Eighteenth-Century Cultural Worlds. The Anatomist and the Fine Arts. London: Routledge, 2018.

20. Gordon RM. The Infamous Burke and Hare. Serial Killers and Resurrectionists in Nineteenth Century Edinburgh.
London: McFarland and Co., 2009.

21. Moore W. The Knife Man. Blood, Body Snatching, and the Birth of Modern Surgery. New York: Broadway Books, 2005.

22. Frank JB. Body Snatching: A Grave Medical Problem. Yale J Biol and Med 1976; 49: 399-410.

23. Burrell S, Gill G. The Liverpool Cholera Epidemic of 1832 and Anatomical Dissection-Medical Mistrust and Civil Unrest. J Hist Med All Sci 2005; 60: 478-98.

24. Berkowitz C. The Illustrious Anatomist: Authorship, Patronage, and Illustrative Style in Anatomy Folios, 1700-1840. Bull Hist Med 2015; 89: 171-208.

25. Kemp M. Style and non-style in Anatomical Illustration: From Renaissance to Henry Gray. J Anat 2010; 216: 192208.

26. Jordanova LJ. Gender, generation and science: William Hunter's obstetrical atlas. En Bynum WF y Porter R. William Hunter and the eighteenth-century medical world. Cambridge: Cambridge University Press, 1985.

27. William Hunter's Tercentenary. University of Glasgow Library. https://universityofglasgowlibrary. wordpress.com/2018/05/23/william-hunters- HYPERLINK "https://universityofglasgowlibrary.wordpress. com/2018/05/23/william-hunters-\%20tercentenary/"tercentenary HYPERLINK "https://universityofglasgowlibrary.wordpress.com/2018/05/23/william-hunters-\%20 tercentenary/"/ (Último acceso el 14 de junio de 2018).

28. Liston JJ. From obstetrics to oryctology: inside the mind of William Hunter (1718-1783). Geological Society of London, Special Publications 2013; 375: 349-73.

29. Porter R. English Society in the 18th Century. London: Penguin, 1991.

30. Obituary William Hunter. Gentleman's Magazine 1783; LIII: 364-6.

31. Fox RH. William Hunter, Anatomist, Physician, Obstetrician (1718-17983), with Notices of his friends Cullen, Smellie, Fothergill, and Baillie. London: Lewis, 1901.

32. Peachey GC. A Memoir of William and John Hunter. Plymouth: William Brendon \& Son, 1924.

33. Handley WS. Makers of John Hunter. Lancet 1939; 1: 369-75.

34. Jacyna LS. Images of John Hunter in the Nineteenth Century. Hist Sci 1983; 21: 85-108.

35. Blizard W. The Hunterian Oration 1815. Royal College of Surgeons, London: Bayley, 1815.

36. Keppie L. William Hunter and the Hunterian Museum in Glasgow 1807-2007. Edinburgh: Edinburgh University Press, 2010. 